\title{
Geoökologische Kartierung als Grundlage für die Bewertung von Funktionen des Landschaftshaushaltes
}

\section{Wozu eine Geoökologische Karte 1 : 25000 ?}

Der Bedarf an raumbezogenen Informationen über den Naturhaushalt ist heute groß und wird noch zunehmen. Im Rahmen von Umweltverträglichkeitsprüfungen, aber auch in der Raumplanung, ist die Berücksichtigung naturhaushaltlicher Gegebenheiten von zentraler Bedeutung. Diese Erkenntnis ist nicht neu, und auch der Begriff der «ökologischen Planung» kommt bereits allmählich in die Jahre. Trotzdem bereitet es immer noch Schwierigkeiten, einzelne oder erst recht komplexe ökologische $\mathrm{Ge}$ gebenheiten im Rahmen der praktischen Arbeit fundiert zu berücksichtigen. Es gibt dafür eine Reihe von Gründen:

1. Die notwendigen Informationen über naturhaushaltliche Gegebenheiten fehlen oder die verfügbaren ökologischen Informationen sind zu separativ und stehen nicht flächendeckend und damit für ein zu bearbeitendes Gebiet nur zufällig zur Verfügung.

2. Die verfügbaren ökologischen Informationen sind nicht "praxislesbar» aufbereitet.

3. Die verfügbaren ökologischen Informationen sind zu weit verstreut und aus Gründen unterschiedlicher Erhebung und Klassifizierung nicht kompatibel.

4. Einfache Methoden zur Erhebung und Ableitung von Geoökosystemparametern und -funktionen existieren zwar teilweise. Sie sind jedoch nirgends zusammengestellt und ungenügend «normiert».

5. Es ist meist schon schwierig, sich im Rahmen einer bestimmten Problemstellung (z. B. UVP Auftrag) einen Überblick über die ökologische Raumsituation zu verschaffen, weil Grundlagen fehlen oder vorhandene Daten nicht verknüpft sind.

Es besteht also zweifellos ein Bedarf nach

1. geeigneten, ausreichend vereinfachten und vereinheitlichten Methoden/Arbeitstechniken, um fehlende geoökologische Einzelinformationen nach Bedarf und Problemstellung zu erfassen (z. B. als Grundlage für Einzelbewertungen) und

2. zusammengefaßten und syntheseartig aufbereiteten, flächendeckenden ökologischen Detailinformationen, die
- einen Überblick über die naturhaushaltliche Raumsituation geben,

- ökologisch wichtige Elemente, Parameter und Prozesse der Landschaft zusammengefaßt darstellen und zueinander in Beziehung setzen,

- Zusammenhänge zwischen Struktur (z. B. Bodenaufbau) und Prozessen erkennen lassen und damit Grundlagen für Abschätzungen landschaftshaushaltlicher Prozesse bereithalten und

- als einheitliche Grundlagen für Bewertungen des landschaftshaushaltlichen Leistungsvermögens (Naturraumpotential) dienen können.

Die Bereitstellung von Daten über naturhaushaltliche Gegebenheiten kann mit einer vereinheitlichten und systematisierten geoökologischen Kartierung einen wesentlichen Schritt vorangetrieben werden. Aus diesem Grund bildete sich 1984 unter der Leitung von H. LESER ein Arbeitskreis «Geoökologische Raumgliederung und landschaftshaushaltliches Leistungsvermögen (Naturraumpotential)». Vertreter geoökologisch arbeitender Hochschulinstitute und der Praxis übernahmen dabei die Aufgabe, ein Handbuch für die Erstellung geoökologischer Karten und eine Anleitung für die einheitliche Ausscheidung der Teilpotentiale des landschaftshaushaltlichen Leistungsvermögens auf der Basis der geoökologischen Kartierung zu entwickeln. Es waren dabei sowohl die im letzten Jahrzehnt in der Geoökosystemforschung (z. B. H. LESER 1983, K. MANNSFELD 1983, TH. MOSIMANN 1984) als auch in der Praxis (z. B. R. MARKS 1982) erarbeiteten Erfahrungen zu berücksichtigen. Die Kartieranleitung liegt nun vor (H. LESER \& H. J. KLINK [Hrsg.] 1988).

Die Kartieranleitung normiert die Erhebung, Übertragung, Ableitung und Klassifizierung der auf Tab. 1 aufgelisteten geoökologischen Einzelparameter und stellt klare Regeln auf für die Aggregierung der Einzelparameter zu Ökotypen. Die auf dieser Basis entstehenden geoökologischen Karten $1: 25000$ (Prinzip und Arbeitsschritte siehe Abb. 1)

Thomas Mosimann, Prof., Dr.,

Geographisches Institut der Universität Hannover, Schneiderberg 50, D-3000 Hannover 1 
- stellen wichtige landschaftshaushaltliche Gegebenheiten zusammenfassend dar,

- grenzen elementare Raumeinheiten (Ökotope) $a b$, für die mit den Begleittabellen (Ökotoptyptabellen) vielseitige Informationen zu Ausstattung und Einzelfunktionen geliefert werden und

- bilden die Grundlage für die Erstellung von "Auswertekarten" der Teilpotentiale des landschaftshaushaltlichen Leistungsvermögens.

\section{Das Konzept der Geoökologischen Karte 1 : 25000 (GÖK 25)}

\subsection{Prinzip und Arbeitsschritte}

Abb. 1 stellt das Prinzip der GÖK 25 und die Hauptarbeitsschritte in einer allgemeinen Übersicht dar. Die Abb. soll auch zeigen, welche «Werkzeuge» die Kartieranleitung für die einzelnen Teilschritte liefert und auf welchen Ebenen vorhandene Daten aus anderen Bereichen einfließen.

Es lassen sich fünf Hauptarbeitsschritte unterscheiden. Die Ausarbeitung des Endergebnisses geschieht mit Schritt vier und fünf. Das Endergebnis besteht aus einer Karte, einem Erläuterungsheft und den voraussichtlich umfangreichen Ökotoptyptabellen als Bestandteil des Erläuterungsheftes. Die zur Karte mitgelieferten Unterlagen erfüllen die Funktion eines geoökologischen Nachschlagewerkes für den erfaßten Raumausschnitt und den betreffenden Landschaftstyp.

Die handbuchartige Kartieranleitung kann auch für die Bearbeitung von Einzelproblemen angewendet werden. In diesem Falle beschränkt man sich auf die Erhebung, Klassifizierung und Aggregierung der Parameter, die für die Bewertung einer einzelnen Teilfunktion des landschaftshaushaltlichen Leistungsvermögens notwendig sind.

\subsection{Die ausgewählten Geoökosystemparameter und ihre Erfassung}

Auf Tab. 1 sind die zu bearbeitenden Geoökosystemparameter zusammengestellt. Je nach Sachund Datenlage sowie Parameter werden diese GröBen

- in der Feldkartierung 1:10000 direkt aufgenommen,

- aus einer bestehenden Kartierung (oder vereinzelt auch aus Luftbildern) übernommen,

- aus flächenhaft erhobenen Ausgangsgrößen abgeleitet (gilt z. B. für die nutzbare Feldkapazität, Einstrahlung usw.) undloder

- aus Standortsaufnahmen nach üblichen Verfahren auf die Fläche extrapoliert (gilt z. B. für Säurestufe, Nährstoffangebot usw.).

Um die Vergleichbarkeit der Aufnahme zu sichern und gleichzeitig der Vielfalt der Landschaftstypen gerecht zu werden, unterscheidet der Parameterkatalog Größen, die immer aufgenommen werden müssen (Minimalkatalog) und andere, die wegen ihrer unterschiedlichen Bedeutsamkeit in verschiedenen Landschaften nur in bestimmten Landschaftstypen bearbeitet werden müssen oder die nach dem räumlichen Auftreten oder bei guter Datenlage mit hinzugenommen werden. Das Konzept ist also außerhalb des Minimalumfangs an Größen flexibel. Es bleibt den Bearbeitern überlassen, bei sachlicher Erfordernis und Möglichkeit der Aufnahme weitere Größen hinzuzunehmen.

$\mathrm{Zu}$ den kartierten bzw. flächenhaft bearbeiteten Geoökosystemparametern kommen bei der Bearbeitung der Prozeßgrößen im Rahmen der Ökotopausscheidung und - «beschreibung» noch einige Klimaparameter hinzu, die für die Berechnung des Lufthaushaltes sowie der Wasser- und Nährstoffversorgungsstufen benötigt werden (siehe dazu z. B. Abb. 2).

Die Erfassung der Parameter ist in der Kartieranleitung Schritt für Schritt beschrieben. Besonders wird dabei auf die Übertragung und Verwertbarkeit bestehender Daten und auf Probleme bei der flächenhaften Aufnahme einzelner Parameter eingegangen. Die Kartieranleitung schlägt in vielen Fällen keine neuen Arbeitstechniken vor, sondern kombiniert bewährte Techniken, auf die wegen der allgemeinen Bekanntheit $z$. T. auch nur verwiesen wird. Entscheidende Punkte sind die Klassifizierung der Parameter für geoökologische Zwecke und für die spätere Ausscheidung der Funktionen des landschaftshaushaltlichen Leistungsvermögens und die Vorschriften für die Ableitung nicht kartierbarer Größen aus verfügbaren Ausgangsparametern (z. B. Bestimmung der nutzbaren Feldkapazität aus Bodenart, Skelettgehalt und Gründigkeit). Aus Umfangsgründen kann hier nicht weiter auf Einzelheiten eingetreten werden.

\subsection{Aggregierung der Geoökosystemparameter zu Ökotopen}

Konzepte und Vorgehensweisen für die Ausscheidung von Ökotopen als landschaftsökologische Grundeinheiten wurden von Praxisseite wegen der mangelnden Anwendbarkeit der Ökotop/Physiotopkarten immer wieder kritisiert. Diese Kritik ist aus zwei Gründen berechtigt:

1. Wegen der uneinheitlichen Verwendung verschiedener Kombinationen von Ausscheidungskriterien waren bisher Ökotoptypen aus verschiedenen Gebieten praktisch nicht vergleichbar und deshalb auch praxisrelevante Informationen nicht systematisch ableitbar.

2. Eine zu allgemeine inhaltliche Charakterisierung mit Hilfe von Komplexgrößen wie Bodenform, Vegetationstyp usw. oder eine zu selektive 
- Informationen über die Bedeutung und Funktion von Einzelparametern

- Vorschriften für die Geländeaufnahme

- Parameterklassifikation

- Hilfstabellen/Diagramme

- Signaturenkatalog

- Hinweise auf methodische und andere

weiterführende Literatur

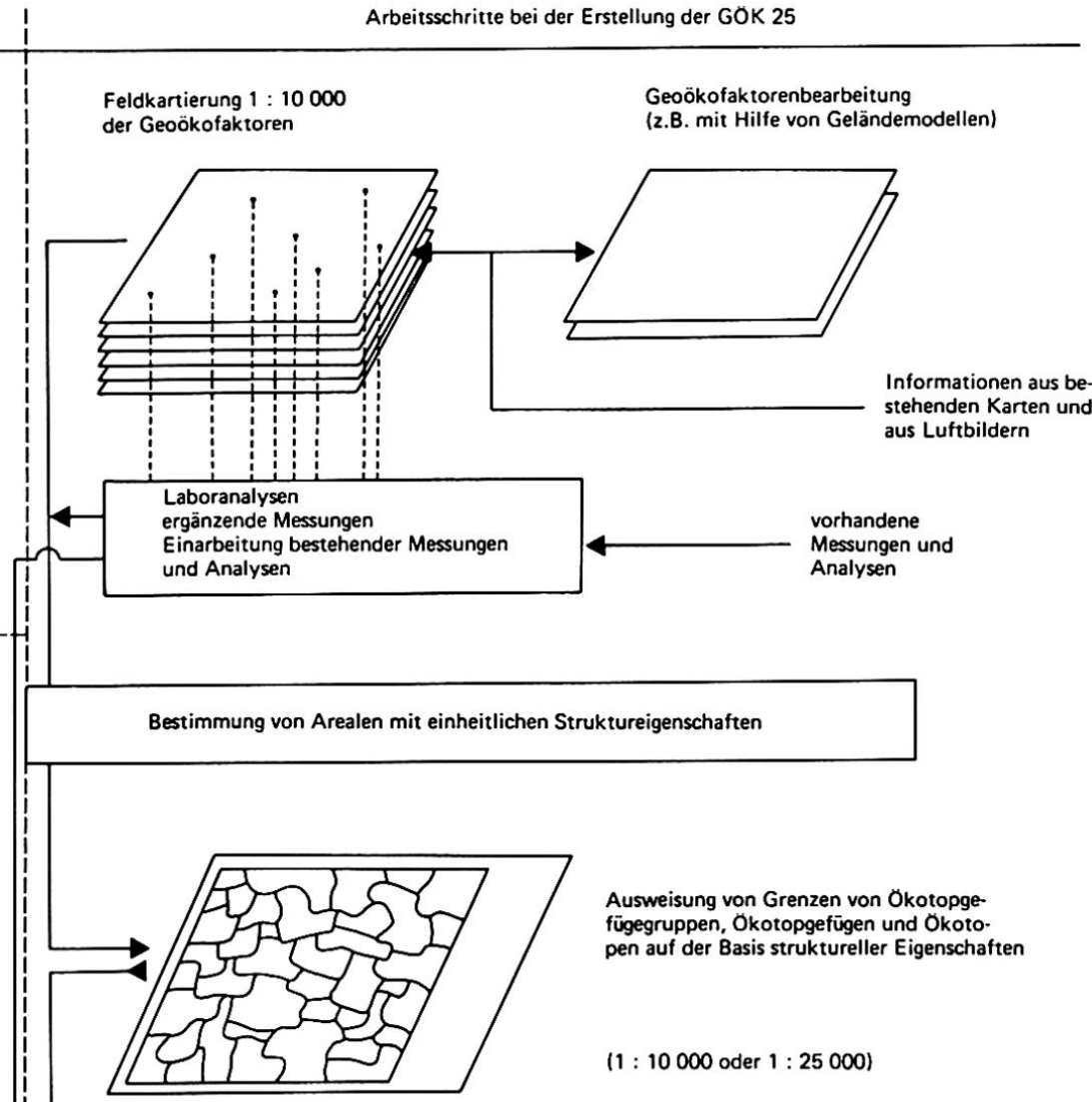

Vorschriften zur Ermittlung von Prozes kennwertstufen in Form von Entscheidungsleitern

Prozessgrössen:

Energiedargebo

Lufthaushalt

Nährstoffhaushalt

Feststofftransport biotische Aktivität Symbolschlüssel Okotope

Signaturenkatalog

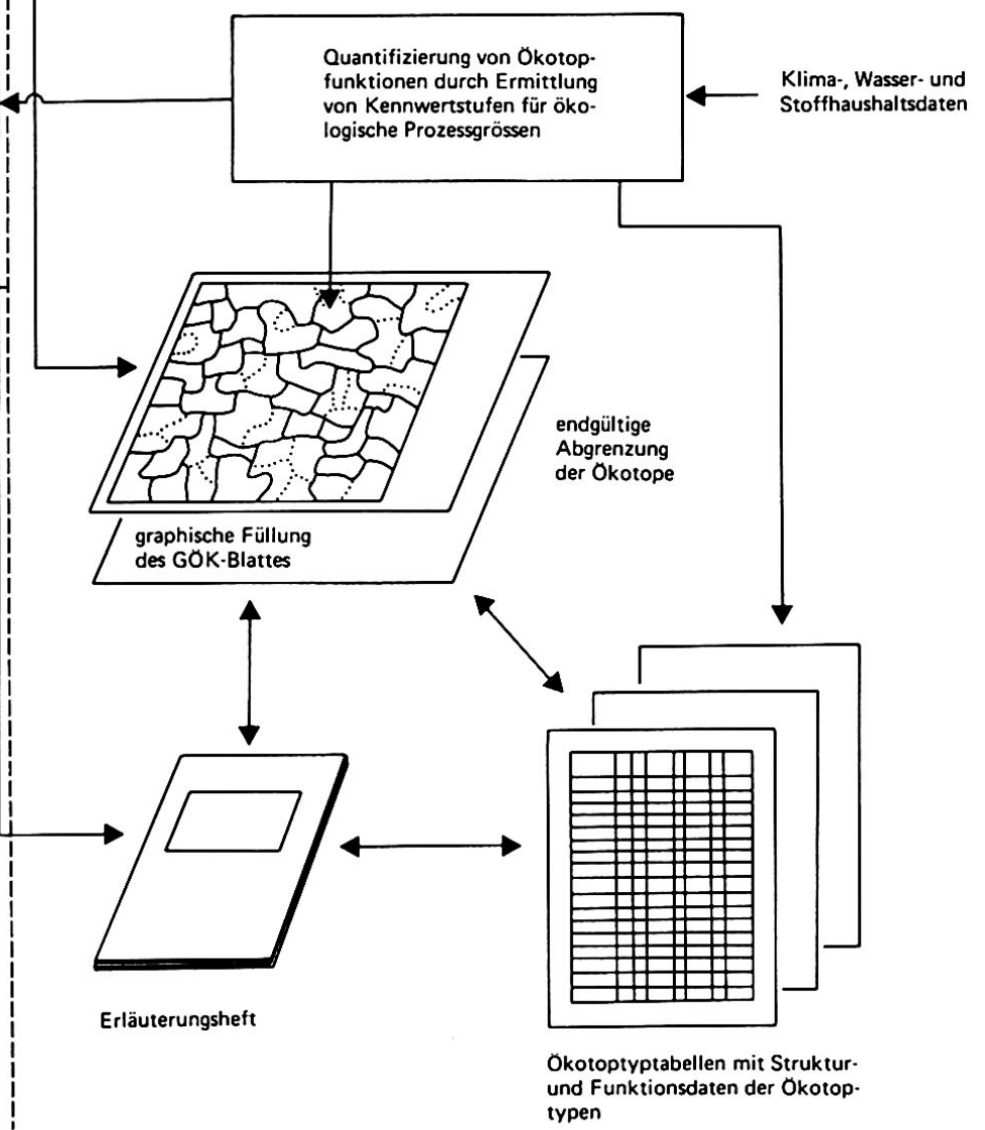

Abb. 1 Prinzip, Arbeitsschritte und Arbeitsergebnisse der Geoökologischen Karte 1:25000

Die bearbeiteten Parameter können Tab. 1 entnommen werden.

(Unter Verwendung eines Schemas aus H. LESER \& H. J. KLINK 1988) 


\begin{tabular}{|c|c|c|c|}
\hline Bereich & $\begin{array}{l}\text { Hauptparameter (="Minimalkatalog") } \\
\text { vollständige Aufnahme bzw. Bear- } \\
\text { beitung in allen Landschaftstypen }\end{array}$ & $\begin{array}{l}\text { zusätzliche Parameter } \\
\text { - die nicht flächendeckend aufge- } \\
\text { nommen werden müssen oder } \\
\text { - die nur in bestimmten Landschafts- } \\
\text { typen aufgenommen werden müssen oder } \\
\text { - je nach Sachlage, Datenlage und Er- } \\
\text { fordernissen einer verfeinerten } \\
\text { Aussage aufgenommen werden können }\end{array}$ & $\begin{array}{l}\text { Komplexgrössen } \\
\text { - die die standörtliche und răum- } \\
\text { liche Aussage ergänzen und } \\
\text { - die ökotopgliederung unter- } \\
\text { stützen können }\end{array}$ \\
\hline Relief & $\begin{array}{l}\text { NEIGUNG } \\
\text { STUFEN UND KANTEN } \\
\text { KLEINE TALER UND TIEFENLINIEN } \\
\text { AKTUELLE GEOMORPHO- } \\
\text { LOGISCHE PROZESSE } \\
\end{array}$ & $\begin{array}{l}\text { WÖLBUNGEN } \\
\text { VOLL- UND HOHLFORMEN } \\
\text { RAUHIGKEI ISFORMEN }\end{array}$ & \\
\hline Boden & $\begin{array}{l}\text { BODENART (INKL. SCHICIITUNG) } \\
\text { Skelettanteil } \\
\text { Humusgehalt } \\
\text { HUMUSFORM } \\
\text { Säurestufe } \\
\text { GRÜNDIGKEIT }\end{array}$ & $\begin{array}{l}\text { Nährstof fangebot } \\
\text { Luf tkapazität }\end{array}$ & Bodenform \\
\hline $\begin{array}{l}\text { Boden- } \\
\text { wasser }\end{array}$ & $\begin{array}{l}\text { GRUNDHASSERFLURABSTAND } \\
\text { STAU-/HANGNÖSSEGRAD } \\
\text { nutzbare Feldkapazităt } \\
\text { Wasserdurchlässigkeit }\end{array}$ & & $\begin{array}{l}\text { ökologischer Feuchtegrad } \\
\text { Bodenfeuchteregimetyp }\end{array}$ \\
\hline $\begin{array}{l}\text { Oberflächen- } \\
\text { gewässer }\end{array}$ & HYDROGEOGRAPHISCHE MERKMALE & & \\
\hline $\begin{array}{l}\text { Gesteins- } \\
\text { untergrund }\end{array}$ & & GESTEINSTYP & \\
\hline Klima & $\begin{array}{l}\text { POTENTIELLE DIREKIE EINSTRAHLUNG } \\
\text { Kaltluf thaushalt } \\
\text { allalla }\end{array}$ & Wind und Durchlüftung & \\
\hline $\begin{array}{l}\text { reale } \\
\text { Vegetation }\end{array}$ & $\begin{array}{l}\text { VEGETATIONSTYP } \\
\text { STRUKTURVIELFALT (Schichtung, } \\
\text { Deckung, Bestandshöhe) }\end{array}$ & $\begin{array}{l}\text { NAIÜRL ICIIKE I ISGRAD } \\
\text { BESTANDSSCHÏDIGUNG }\end{array}$ & PFLANZENGESELLSCIUATEN \\
\hline $\begin{array}{l}\text { anthropo- } \\
\text { gene Ein- } \\
\text { flüsse }\end{array}$ & $\begin{array}{l}\text { ABGRENZUNG ANTHROPOGEN } \\
\text { ÜBERFORMTER BEREICHE }\end{array}$ & $\begin{array}{l}\text { ÖKOLOGISCHE CHARAKTERISIERUNG ANTHROPO- } \\
\text { GEN ÜBERFORIITER BEREICHE }\end{array}$ & \\
\hline
\end{tabular}

Tab. 1 Im Rahmen der Geoökologischen Karte 1:25000 kartierte und bearbeitete Ökosystem-Parameter und Komplexgrößen

\section{BODENART}

\section{Säurestufe}

Nutzbare

Feldkapazität

Wind und Durchlüftung
= Parameter, die flächendeckend aufgenommen werden müssen (Geländeaufnahme), Übernahme aus bestehenden Karten, teilweise Übernahme aus Luftbildern, Ableitung aus Reliefdaten (Geländemodell).

= Parameter, die aus Leitprofilaufnahmen (einschließlich Laboranalysen) auf die zugehörigen Bodenareale extrapoliert werden.

= Parameter, die aus Strukturmerkmalen abgeleitet, z. T. unter Beizug von Laboranalysen und vorhandenen Messungen, und entsprechenden Arealen mit einheitlichen strukturellen Gegebenheiten zugeordnet werden.

= Parameter, die nach einer Messung an Standardpunkten oder Standardcatenen auf Areale mit jeweils entsprechenden strukturellen Gegebenheiten extrapoliert werden.

Die Kartieranleitung beschreibt für jeden einzelnen Parameter: Funktion im Geoökosystem, Erhebung, Klassifizierung und besondere Aufnahmeprobleme. 


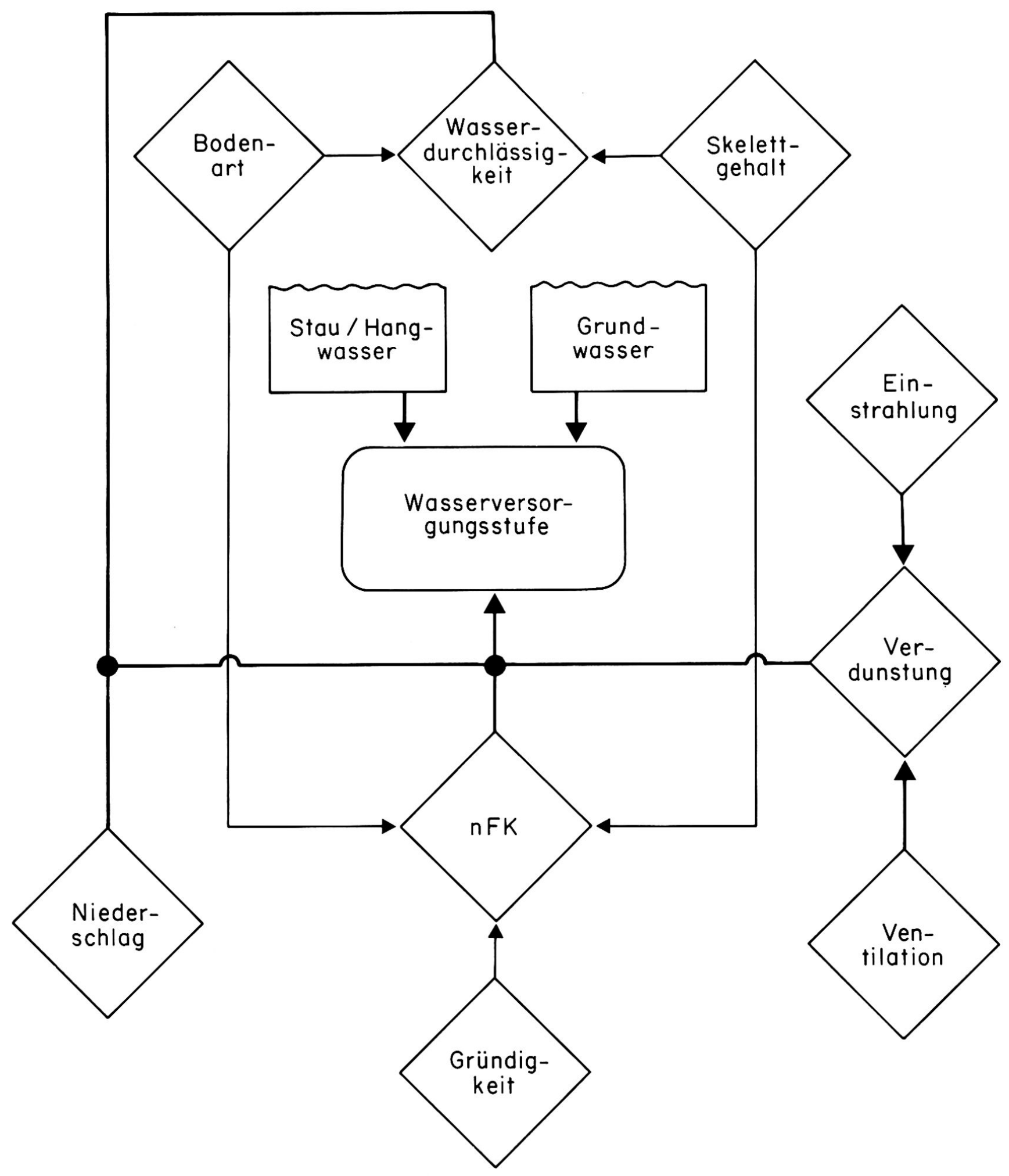

Abb. 2 Das Prinzip der Verknüpfung von Struktur- und Prozeßgrößen zur funktionalen Charakterisierung der Ökotope - Beispiel Wasserversorgung

Ein analoges Vorgehen wird für die Bestimmung von Energiedargebot, Lufthaushalt, Feststofftransport, Nährstoffhaushalt und biotische Aktivität angewandt. Da die jeweils berücksichtigten Regler und Prozeßgrößen zum Teil auch untereinander verknüpft sind, umfaßt die inhaltliche Kennzeichnung der Ökotope ein ganzes Netzwerk von Struktur- und Prozeßgrößen. 
Auswahl der für die Ausscheidung verwendeten Parameter schuf keinen genügend eindeutigen Zusammenhang zwischen Ökotoptyp und seinen ökologischen Einzeleigenschaften. Der Ökotoptyp war damit als Bezugsfläche für praxisorientierte Einzelbewertungen zuwenig genau definiert.

Um diese Schwächen zu beseitigen, wurde das Konzept für die Ökotopausscheidung in der GÖK 25 wie folgt aufgebaut:

1. Die Ausscheidung geschieht streng induktiv, basierend auf eindeutig definierten bzw. klassifizierten Einzelparametern.

2. Die Ökotopausscheidung basiert auf einem «Verknüpfungsfeld» von teilweise unabhängigen, teilweise gegenseitig abhängigen Struktur- und Prozeßgrößen. Abb. 2 soll dieses Prinzip für den Teilbereich Wasserversorgung exemplarisch veranschaulichen. Es fließen dabei direkt und indirekt bei der Bestimmung von Prozeßgrößenstufen gesamthaft rund 30 Einzelparameter in die Ökotopausgliederung und den Ökotopbeschrieb ein. Mit diesem «Modell» soll eine dem heutigen Kenntnisstand entsprechende Annäherung an die komplexe ökologische Wirklichkeit erreicht werden.

3. Die Ausscheidung und Charakterisierung der Ökotope geschieht in zwei Schritten (siehe dazu Abb. 1). Eine erste Gliederung in Raumeinheiten wird auf der Basis von elf Strukturgrößen (siehe Abb. 1) erreicht. Die Klassen der Strukturgrößen sind dabei relativ weit gehalten, damit der Strukturgrößenraster für möglichst alle Landschaftstypen angewendet werden kann. In einem zweiten Schritt erfolgt mit Hilfe der Prozeßgrößenbereiche die definitive Charakterisierung der Ökotope. Bei der Ausscheidung der Prozeßkennwertstufen fließen dabei weitere wichtige Parameter mit Reglerfunktion in die Ökotopcharakterisierung mit ein (siehe dazu ebenfalls Beispiel «Wasserversorgungsstufe» auf Abb. 2).

Alle Einzelmerkmale des geoökologischen Struktur- und Prozeßbereiches werden für alle Ökotoptypen auf Tabellen zusammengestellt. Eine ergänzende Beurteilung des voraussichtlichen ökologischen Verhaltens der einzelnen Raumeinheiten bei bestimmten Nutzungen oder Eingriffen kann also jeweils durchgeführt werden.

\section{Bewertung des Leistungsvermögens des Landschaftshaushaltes auf der Basis der Geoökolo- gischen Karte 1 : 25000}

Die bisher geschilderte GÖK 25 stellt Grundlagendaten für den Anwender bereit. Dabei werden geoökologisch relevante Daten in einheitlicher Form erho- ben, verknüpft und dargestellt. Eine vielseitige $D a$ tenressource für die Praxis liegt vor.

Auf dieser einheitlichen Datenbasis erfolgt nun die Bewertung der Teilfunktionen des Leistungsvermögens des Landschaftshaushaltes. Mit diesem Schritt werden für den Anwender auf verschiedenen Planungsebenen direkt benützbare "Auswertungskarten» bereitgestellt.

Der Arbeitskreis arbeitet das Handbuch für die $\mathrm{Be}-$ wertung im Moment aus (= Bewertungsanleitung Leistungsvermögen Landschaftshaushalt). Dabei werden bestehende Bewertungsverfahren zusammengezogen und auf die einheitliche Datenbasis angepa $B t$ wie auch neue Bewertungsverfahren entwikkelt. Es erfolgt zum Teil auch eine Diskussion und Neufassung der verschiedenen Leistungsbereiche bzw. Teilfunktionen des landschaftshaushaltlichen Leistungsvermögens.

Die handbuchartige Bewertungsanleitung wird folgende Teilfunktionen (und Potentiale) umfassen:

- Ökotopbildungsfunktion,

- Erosionsschutzfunktion,

- Filter-, Puffer- und Transformatorfunktion des Bodens,

- Grundwasserschutzfunktion,

- Grundwasserneubildungsfunktion,

- Abflußregulationsfunktion,

- Luftregenerationsfunktion,

- Lärmschutzfunktion,

- Klimameliorationsfunktion,

- Land- und forstwirtschaftliches Ertragspotential,

- Wasserdargebotspotential (Oberflächenwasserund Grundwasserdargebot),

- Erholungsfunktion.

\section{Zusammengefaßte Aspekte zum Konzept der GÖK 25 im Hinblick auf die Anwendung}

1. Die geoökologischen Grundlagen werden mit der GÖK 25 so aufbereitet, daß sie direkt für Bewertungen übernommen werden können. Die überregionale Vergleichbarkeit ist durch Standardisierung gesichert.

2. Das Verfahren der Charkaterisierung von Geoökosystemen wird transparent und nachvollziehbar. Rückschlüsse auf einzelne ökologische Eigenschaften sind deshalb auf allen Bearbeitungsstufen möglich. Aus den Ökotoptyptabellen wird man direkt entnehmen können, welche Ökotope bestimmte gesuchte Einzeleigenschaften aufweisen und mit welcher Bandbreite weitere wichtige ökologische Eigenschaften in einer Gruppe bestimmter Ökotoptypen auftreten.

3. Das Konzept der Ökotopausscheidung läßt eine verfeinerte Gliederung nach einzelnen Parametern im Rahmen besonderer Problemstellungen offen (z. B. großmaßstabige Bewertungen im Rahmen 
der Nutzungsplanung). Hier muß der Anwender zwar eigene Arbeit leisten. Eine Zeitersparnis ergibt sich aber daraus, daß viel Information standarisiert mitgeliefert wird und die Karte nach dem Baukastenprinzip aufgebaut ist.

4. Die Kartieranleitung stellt ein Nachschlagewerk dar für die praktische Bearbeitung verschiedenster landschaftsökologischer Einzelprobleme. Sie ist eine Verfahrenssammlung und liefert auch Aussagen über die Verwertbarkeit von Einzelparametern im Rahmen von Bewertungen und gutachterischer Arbeit. Die Arbeit mit der Kartieranleitung muß nicht zwangsläufig zur Ausscheidung von Ökotopen führen. Sofern für Spezialfragestellungen nur ein Teil der Parameter für eine Bewertung benötigt werden, beschränkt sich der Benutzer auf diese.

\section{Zusammenfassung}

Im Arbeitskreis «Geoökologische Raumgliederung und landschaftshaushaltliches Leistungsvermögen» wurde in den letzten drei Jahren ein Handbuch zur vereinfachten und vereinheitlichten Erfassung geoökologischer Parameter (siehe Tab. 1) und zur Erstellung einer geoökologischen Karte 1:25000 (GÖK 25) entwickelt. Diese geoökologischen Karten stellen wichtige landschaftshaushaltliche Gegebenheiten zusammenfassend dar und grenzen Ökotope ab, für die Ökotoptyptabellen detaillierte Einzelinformationen liefern. Die Karten bilden die Grundlage für die Ausscheidung der Teilfunktionen/Teilpotentiale des landschaftshaushaltlichen Leistungsvermögens.
Abb. 1 stellt das Prinzip und die fünf Hauptarbeitsschritte der GÖK 25 dar. Die ausgewählten Geoökosystemparameter faßt Tab. 1 zusammen. Die Art und Weise ihrer Aufnahme und Aggregierung zu Ökotoptypen wird kurz diskutiert. Auf der Basis der durch die GÖK 25 bereitgestellten praxisrelevanten Grundlagendaten erfolgt die Bewertung der in Kap. 3 aufgelisteten haushaltlichen Teilfunktionen. Auf weitere Anwendungsaspekte von GÖK 25 und Handbuch weist das abschließende Kapitel hin.

\section{Literatur}

LESER, H. (1983): Geoökologie: Probleme, Möglichkeiten und Grenzen geoökologischer Arbeit heute. In: Geographische Rundschau, 35, S. 212-221.

LESER, H. (1988): Die GÖK 25: Konzept und Anwendungsperspektiven der Geoökologischen Karte 1:25000. In: Geographische Rundschau, 40, H. 5.

LESER, H. \& KLINK, H.-J. (Hrsg.) (1988): Handbuch und Kartieranleitung Geoökologische Karte 1:25000 (KA GÖK 25). Bearbeitet vom Arbeitskreis "Geoökologische Karte und Naturraumpotential" des Zentralausschusses für deutsche Landeskunde. Forsch. z. deutschen Landeskunde, Bd. 228, Trier.

MANNSFELD, K. (1983): Landschaftsanalyse und Ableitung von Naturraumpotentialen. Abh. Sächs. Akad. Wiss. zu Leipzig, Math.-nat. KI., Bd. 55, H. 3, Berlin.

MARKS, R. (1982): Methodik der Analyse und Bewertung von Teilkomponenten des Naturhaushaltes im Rahmen der Landschaftsplanung, Essen.

MOSIMANN, TH. (1984): Landschaftsökologische Komplexanalyse, Wiesbaden. 